\title{
Solving fuzzy bi-matrix games through a interval value function approach
}

\author{
Kaisheng Liu $^{1}$, Yumei Xing ${ }^{2 *}$ \\ ${ }^{1}$ School of Mathematics and Statistics, Tianshui Normal University, \\ Tianshui 741001, China \\ ${ }^{2}$ College of Science, Chongqing University of Post and Telecommunication, \\ Chongqing, 400065, China
}

\begin{abstract}
This article puts forward the bi-matrix games with crisp parametric payoffs based on interval value function approach. We conclude that the equilibrium solution of the game model can converted into optimal solutions of the pair of the non-linear optimization problem. Finally, experiment results show the efficiency of the model.

Keywords: fuzzy bi-matrix game; equilibrium solution; non-linear optimization problem
\end{abstract}

\section{Introduction}

Generally speaking, the bi-matrix game model is accurate, when the bet is a small amount of money. In real-life, the relations between the interests of people are more complex. Particularly, in some areas of the economy, the interests of the two players is precisely the opposite. It is well known that these games are two person non-zero-sum game, which are called bi-matrix games for short. Therefore, much research in recent years has focus on bi-matrix game problems.

Mangasarian et al.[21] showed that the equilibrium solution of bi-matrix game is equivalent to the optimization problem about linear constraints and a quadratic objective function. Chakeri et al. [8] used fuzzy logic to determine the priority of the payoff based on the linguistic preference relation and proposed the notion of linguistic Nash equilibriums. Fuzzy preference relation has been widely used in the fuzzy game theory $[5,6,7,9]$. At the same time, they [9] utilize the same method [8] to determine the priority of the payoff based on fuzzy preference relation. In order to deal with this game model, a new approach was put forward. Moreover, Sharifian et al. [27] also applied fuzzy linguistic preference relation to the the fuzzy game theory. Hladik [15]

\footnotetext{
${ }^{*}$ Corresponding author. Tel.:+86-13364008435; E-mail: x-ym2015@163com (Y.M. Xing).
} 
introduced interval bi-matrix games and obtained the conclusion that the equilibrium solution of interval bi-matrix game can be changed into the linear mixed integer optimization problem. The methods of solving the interval bi-matrix games by a pair of auxiliary bilinear programming models is discussed by Fei and Li [16].

There have been a great number of studies in single objective fuzzy bi-matrix games $[2,17,20,31,32,33]$ and intuitionistic fuzzy bi-matrix games [18, 22, 23]. Maeda [20] discussed a bi-matrix game with fuzzy payoffs and proved the existence of Nash equilibrium in a fuzzy bi-matrix game. Vidyottama et al. [31] confirmed that a bimatrix game with fuzzy goal and a crisp non-linear programming problem are of equal value. Vijay et al.[32] presented a bi-matrix game with fuzzy payoffs and fuzzy goals through a fuzzy relation method, and they showed that the solution is equal to two semi-infinite optimization problems. Li [17] proposed the concept of crisp parametric bi-matrix games and discussed the existence of equilibrium solutions of fuzzy bi-matrix games. Recently, Nayak and Pal [23] studied a bi-matrix game with intutionistic fuzzy goals. Subsequently, Li [18] discussed the way to deal with the bi- matrix games with payoffs of intuitionistic fuzzy sets via bilinear programming method and defuzzification ranking approach.

Although the research of bi-matrix game problems have been extensively studied in recent years, it needs to be further conducted from the perspective of the non-linear optimization approach. Taking elicitation from $[2,16,18]$, we can take inspiration and put forward a non-linear optimization method for the bi-matrix games with crisp parametric payoffs based on interval value function in this paper.

The outline of this article is as follows: Section 2 describes the basic definitions and recalls some results concerning fuzzy numbers and the crisp single-objective bimatrix game. In section 3, the bi-matrix games with crisp parametric payoffs based on interval value function approach is presented. We conclude that the equilibrium solution of the game model can converted into the pair of optimal solutions of the non-linear optimization problem. In section 4 , an example is provided to illustrate the efficiency of the model in this paper. Conclusions and suggestions for future research are given in the last section.

\section{Preliminaries}

The following notations, definitions and results will be needed in the sequel.

We denote $\mathcal{K}_{C}$ as the family of all bounded closed intervals in $\mathbb{R}$ [13], that is,

$$
\mathcal{K}_{C}=\left\{\left[a_{L}, a_{R}\right] \mid a_{L}, a_{R} \in \mathbb{R} \text { and } a_{L} \leq a_{R}\right\} .
$$

A fuzzy set $\widetilde{x}$ of $\mathbb{R}$ is characterized by a membership function $\mu_{\widetilde{x}}: \mathbb{R} \rightarrow[0,1]$. For each such fuzzy set $\widetilde{x}$, we denote by $[\widetilde{x}]^{\alpha}=\left\{x \in \mathbb{R}: \mu_{\widetilde{x}}(x) \geq \alpha\right\}$ for any $\alpha \in(0,1]$, its $\alpha$-level set. Define the set $[\widetilde{x}]^{0}$ by $[\widetilde{x}]^{0}=\overline{\bigcup_{\alpha \in(0,1]}[\widetilde{x}]^{\alpha}}$, where $\bar{A}$ denotes the closure of a crisp set $A$. A fuzzy number $\widetilde{x}$ is a fuzzy set with non-empty bounded closed level sets $[\widetilde{x}]^{\alpha}=\left[\widetilde{x}_{L}(\alpha), \widetilde{x}_{R}(\alpha)\right]$ for all $\alpha \in[0,1]$, where $\left[\widetilde{x}_{L}(\alpha), \widetilde{x}_{R}(\alpha)\right]$ denotes a closed interval 
with the left end point $\widetilde{x}_{L}(\alpha)$ and the right end point $\widetilde{x}_{R}(\alpha)[14]$. We denote the class of fuzzy numbers by $\mathscr{F}$.

Definition 1 [30] Suppose that $\widetilde{x}$ and $\widetilde{y}$ are two fuzzy numbers. Then $\widetilde{x}$ precedes $\widetilde{y}$ $(\widetilde{x} \preceq \widetilde{y})$ if and only if $[u]^{\alpha}=\left[u_{L}(\alpha), u_{R}(\alpha)\right] \leq[v]^{\alpha}=\left[u_{L}(\alpha), u_{R}(\alpha)\right]$ for each $\alpha \in[0,1]$, where $[u]^{\alpha} \leq[v]^{\alpha}$ if and only if $u_{L}(\alpha) \leq v_{L}(\alpha)$ and $u_{R}(\alpha) \leq v_{R}(\alpha)$.

Note that the order relation $\preceq$ is reflexive and transitive. Moreover, any two elements of $\mathscr{F}$ are comparable under the ordering $\preceq$. For more details see $[28,29]$.

Definition 2 [34] Let $\widetilde{x}$ be fuzzy numbers, If the membership function $u_{\widetilde{x}}(x)$ of the fuzzy number $\widetilde{x}$ is denoted by

$$
u_{\widetilde{x}}(x)=\left\{\begin{array}{lr}
0, & x<\dot{a}, x>\grave{a} \\
\frac{x-\grave{a}}{a-\grave{a}}, & a \leq x \leq a \\
\frac{\grave{a}-x}{\grave{a}-a}, & a<x \leq \grave{a}
\end{array}\right.
$$

Then, $\widetilde{x}$ is called a triangular fuzzy number. And the triangular fuzzy number $\widetilde{x}$ is presented by $\widetilde{x}=(\dot{a}, a, \grave{a})$.

Furthermore, the $\alpha$-level set of the triangular fuzzy number $\widetilde{x}=(\dot{a}, a, \grave{a})$ is the closed interval [34]

$$
[\widetilde{x}]^{\alpha}=\left[\widetilde{x}_{L}(\alpha), \widetilde{x}_{R}(\alpha)\right]=[(a-\grave{a}) \alpha+\grave{a},-(\grave{a}-a) \alpha+\grave{a}], \quad \alpha \in(0,1] .
$$

Definition 3 [19] Let $\widetilde{a}_{i}$ be fuzzy numbers and $x_{i} \geq 0(i=1,2, \cdots, n)$ be an real numbers. Then, $\sum_{i=1}^{n} \widetilde{a}_{i} x_{i}$ is a fuzzy numbers.

In the following, we shall describe a crisp bi-matrix game model in $[1,2]$.

Definition 4 [2] The set of mixed strategies for player I is denoted by

$$
S^{m}=\left\{x=\left(x_{1}, x_{2}, \cdots, x_{m}\right)^{T} \in \mathbb{R}^{m} \mid \sum_{i=1}^{m} x_{i}=1, x_{i} \geq 0, i=1,2, \cdots, m .\right\}
$$

Similarly, The set of mixed strategies for player II is denoted by

$$
S^{n}=\left\{y=\left(y_{1}, y_{2}, \cdots, y_{n}\right)^{T} \in \mathbb{R}^{n} \mid \sum_{j=1}^{n} y_{j}=1, y_{j} \geq 0, j=1,2, \cdots, n .\right\}
$$

where $x^{T}$ is the transposition of $x, \mathbb{R}^{m}$ and $\mathbb{R}^{n}$ are $m$ - and $n$-dimensional Euclidean spaces. 

$[1,2]$

A payoff matrix of the Player I and Player II in bi-matrix game are denoted by

$$
A=\left(\begin{array}{ccc}
a_{11} & \cdots & a_{1 n} \\
\vdots & \ddots & \vdots \\
a_{m 1} & \cdots & a_{m n}
\end{array}\right)
$$

and

$$
B=\left(\begin{array}{ccc}
b_{11} & \cdots & b_{1 n} \\
\vdots & \ddots & \vdots \\
b_{m 1} & \cdots & b_{m n}
\end{array}\right)
$$

respectively. Assume that the player I and player II both are maximized players. A bi-matrix game $(B G)$ model in $[2]$ is taken as

$$
B G=\left(S^{m}, S^{n}, A, B\right) .
$$

Definition 5 [1, 2] Let $A$ be a payoff matrix of the Player I. When Player I and Player II choose mixed strategies $x \in S^{m}$ and $y \in S^{n}$, respectively. An expected payoff of Player I is denoted by

$$
E(x, y, A)=x^{T} A y=\sum_{i=1}^{m} \sum_{j=1}^{n} a_{i j} x_{i} y_{j}
$$

Similarly, let B be a payoff matrix of the Player II, an expected payoff of Player II is denoted by

$$
E(x, y, B)=x^{T} B y=\sum_{i=1}^{m} \sum_{j=1}^{n} b_{i j} x_{i} y_{j}
$$

Definition $6[2,16]\left(x^{*}, y^{*}\right) \in S^{m} \times S^{n}$ is called the pair of equilibrium solution of the game $(B G)$ model if

$$
\begin{aligned}
& x^{T} A y^{*} \leq\left(x^{*}\right)^{T} A y^{*}, \forall x \in S^{m}, \\
& \left(x^{*}\right)^{T} B y \leq\left(x^{*}\right)^{T} B y^{*}, \forall y \in S^{n} .
\end{aligned}
$$

Theorem 1 [2, 16] For any a given game $(B G)$ model, $\left(x^{*}, y^{*}\right)$ is an equilibrium solution of game $(B G)$ model if and only if it is an optimal solution the following problem (NLP1) model. 
$(N L P 1)$

$$
\begin{aligned}
\max & \left\{x^{T} A y+x^{T} B y-u-v\right\} \\
\text { subject to } & \sum_{j=1}^{n} a_{i j} y_{j} \leq u(i=1,2, \cdots, m), \\
& \sum_{i=1}^{m} b_{i j} x_{i} \leq v, \quad(j=1,2, \cdots, n), \\
& \sum_{i=1}^{m} x_{i}=1, \\
& \sum_{j=1}^{n} y_{j}=1, \\
& x_{i} \geq 0, y_{j} \geq 0, \\
& u, v \text { unrestricted in sign. }
\end{aligned}
$$

\section{A Bi-matrix Game Model based on Interval Value Func- tion}

In this section, we will establish a bi-matrix game model through a interval value function. Let $S^{m}$ and $S^{n}$ be introduced in section 2. Suppose that the elements of $\widetilde{A}$ and $\widetilde{B}$ be fuzzy numbers. Therefore, the bi-matrix game with fuzzy payoffs [2], denoted by $B G F P$, can be presented as

$$
B G F P=\left(S^{m}, S^{n}, \widetilde{A}, \widetilde{B}\right) .
$$

Definition 7 Let $\widetilde{A}$ be fuzzy payoff matrix of the Player I. When Player I and Player II choose mixed strategies $x \in S^{m}$ and $y \in S^{n}$, respectively. A fuzzy expected payoff of Player I is denoted by

$$
E(x, y, \widetilde{A})=x^{T} \widetilde{A} y=\sum_{i=1}^{m} \sum_{j=1}^{n} \widetilde{a}_{i j} x_{i} y_{j}
$$

Similarly, let $\widetilde{B}$ be fuzzy payoff matrix of the Player II, a fuzzy expected payoff of Player II is denoted by

$$
E(x, y, \widetilde{B})=x^{T} \widetilde{B} y=\sum_{i=1}^{m} \sum_{j=1}^{n} \widetilde{b}_{i j} x_{i} y_{j}
$$

Definition 8 Let $(x, y) \in S^{m} \times S^{n}$. If $x \in S^{m}$ and $y \in S^{n}$ satisfy the following conditions:

$$
\begin{aligned}
& x^{T} \widetilde{A} y^{*} \preceq\left(x^{*}\right)^{T} \widetilde{A} y^{*}, \forall x \in S^{m}, \\
& \left(x^{*}\right)^{T} \widetilde{B} y \preceq\left(x^{*}\right)^{T} \widetilde{B} y^{*}, \forall y \in S^{n} .
\end{aligned}
$$

Then $\left(x^{*}, y^{*}\right) \in S^{m} \times S^{n}$ is called the equilibrium solution of $(B G F P)$. 
In order to get the equilibrium solution of $(B G F P)$, next, we construct crisp parametric payoff matrixes based on interval value function of Player I and Player II as follows:

$$
I(\widetilde{A})=\left(\begin{array}{ccc}
{\left[\left(\widetilde{a}_{11}\right)_{L}(\alpha),\left(\widetilde{a}_{11}\right)_{R}(\alpha)\right]} & \cdots & {\left[\left(\widetilde{a}_{1 n}\right)_{L}(\alpha),\left(\widetilde{a}_{1 n}\right)_{R}(\alpha)\right]} \\
\vdots & \ddots & \vdots \\
{\left[\left(\widetilde{a}_{m 1}\right)_{L}(\alpha),\left(\widetilde{a}_{m 1}\right)_{R}(\alpha)\right]} & \cdots & {\left[\left(\widetilde{a}_{m n}\right)_{L}(\alpha),\left(\widetilde{a}_{m n}\right)_{R}(\alpha)\right]}
\end{array}\right)
$$

and

$$
I(\widetilde{B})=\left(\begin{array}{ccc}
{\left[\left(\widetilde{b}_{11}\right)_{L}(\alpha),\left(\widetilde{b}_{11}\right)_{R}(\alpha)\right]} & \cdots & {\left[\left(\widetilde{b}_{1 n}\right)_{L}(\alpha),\left(\widetilde{b}_{1 n}\right)_{R}(\alpha)\right]} \\
\vdots & \ddots & \vdots \\
\left.\left[\widetilde{b}_{m 1}\right)_{L}(\alpha),\left(\widetilde{b}_{m 1}\right)_{R}(\alpha)\right] & \cdots & {\left[\left(\widetilde{b}_{m n}\right)_{L}(\alpha),\left(\widetilde{b}_{m n}\right)_{R}(\alpha)\right]}
\end{array}\right)
$$

Thus, we obtain that the bi-matrix game based on interval value function, denoted by $B G I V F P$, can be presented as

$$
B G I V F P=\left(S^{m}, S^{n}, I(\widetilde{A}), I(\widetilde{B})\right) .
$$

Next, we give an equivalent definition of equilibrium solution of $(B G F P)$.

Definition 9 Let $\alpha \in[0,1],(x, y) \in S^{m} \times S^{n}$. If $x \in S^{m}$ and $y \in S^{n}$ satisfy following the conditions:

$$
\begin{aligned}
& x^{T} I(\widetilde{A}) y^{*} \preceq\left(x^{*}\right)^{T} I(\widetilde{A}) y^{*}, \forall x \in S^{m}, \\
& \left(x^{*}\right)^{T} I(\widetilde{B}) y \preceq\left(x^{*}\right)^{T} I(\widetilde{B}) y^{*}, \forall y \in S^{n} .
\end{aligned}
$$

Then, $\left(x^{*}, y^{*}\right) \in S^{m} \times S^{n}$ is called the equilibrium solution of $(B G I V F P)$.

Theorem 2 The point $\left(x^{*}, y^{*}\right) \in S^{m} \times S^{n}$ is the equilibrium solution of $(B G F P)$ if and only if it is the equilibrium solution of (BGIVFP).

Proof. Since the elements of $\widetilde{A}$ and $\widetilde{B}$ be fuzzy numbers, by Definition 2, we obtain that the elements of $x^{T} \widetilde{A} y^{*},\left(x^{*}\right)^{T} \widetilde{A} y^{*},\left(x^{*}\right)^{T} \widetilde{B} y$ and $\left(x^{*}\right)^{T} \widetilde{B} y^{*}$ are fuzzy numbers. Using Definition 1, we have

$$
\begin{gathered}
x^{T} \widetilde{A} y^{*} \preceq\left(x^{*}\right)^{T} \widetilde{A} y^{*} \Leftrightarrow x^{T} I(\widetilde{A}) y^{*} \preceq\left(x^{*}\right)^{T} I(\widetilde{A}) y^{*}, \forall x \in S^{m}, \\
\left(x^{*}\right)^{T} \widetilde{B} y \preceq\left(x^{*}\right)^{T} \widetilde{B} y^{*} \Leftrightarrow\left(x^{*}\right)^{T} I(\widetilde{B}) y \preceq\left(x^{*}\right)^{T} I(\widetilde{B}) y^{*}, \forall y \in S^{n} .
\end{gathered}
$$

Thus, The point $\left(x^{*}, y^{*}\right) \in S^{m} \times S^{n}$ is the equilibrium solution of $(B G F P)$ if and only if it is the equilibrium solution of $(B G I V F P)$.

Therefore, we convert the equilibrium solution of $(B G F P)$ to the equilibrium solution of $(B G I V F P)$. Now, we consider the game $(B G I V F P)$ model. By Definition 9, we conclude the following the theorem. 
Theorem $3\left(x^{*}, y^{*}\right) \in S^{m} \times S^{n}$ is the equilibrium solution of $(B G I V F P)$ if only and if it is the optimal solution of the following problems $(P 1)$.

$$
\begin{aligned}
& \max _{x} x^{T} I(\widetilde{A}) y^{*}+\max _{y}\left(x^{*}\right)^{T} I(\widetilde{B}) y \\
& \text { such that } \forall x \in S^{m}, \forall y \in S^{n} .
\end{aligned}
$$

Proof. By Definition 9, we can obtain that the equilibrium solution of (BGIVFP) is equal to the following two problems $(P 2)$ and $(P 3)$.

$$
\begin{aligned}
& \max _{x} x^{T} I(\widetilde{A}) y^{*} \\
& \text { such that } \forall x \in S^{m} .
\end{aligned}
$$

$$
\begin{aligned}
& \max _{y}\left(x^{*}\right)^{T} I(\widetilde{B}) y \\
& \text { such that } \forall y \in S^{n} .
\end{aligned}
$$

In terms of $\forall x \in S^{m}$ and $\forall y \in S^{n}$, the constraints of (P2) and (P3) are separable. Then, the problems $(P 2)$ and $(P 3)$ can be rewritten as $(P 1)$.

$$
\begin{aligned}
& \max _{x} x^{T} I(\widetilde{A}) y^{*}+\max _{y}\left(x^{*}\right)^{T} I(\widetilde{B}) y \\
& \text { such that } \forall x \in S^{m}, \forall y \in S^{n} .
\end{aligned}
$$

Thus, the proof of this theorem is completed.

Based on the above discusses, taking $\left(\widetilde{a}_{i j}, \widetilde{b}_{i j}\right) \in \widetilde{A}_{i j} \times \widetilde{B}_{i j}(i=1,2, \cdots, m ; j=$ $1,2, \cdots, n)$, we consider a fuzzy bi-matrix game $(B G F P)$. In terms of fuzzy bi-matrix game $(B G F P)$, by Definition 8 , it is obvious that the values of Players $I$ and Players $I I$ are functions of $\widetilde{a}_{i j}$ and $\widetilde{b}_{i j}$, respectively. That is, the value $u$ of Players $I$ is a function of values $\widetilde{a}_{i j}$, denoted by $u=u\left(\widetilde{a}_{i j}\right)$. At the same time, $x^{*} \in S^{m}$ of Players $I$ is also a function of values $\widetilde{a}_{i j}$, denoted by $x^{*}=x^{*}\left(\widetilde{a}_{i j}\right)$. Similarly, the value $v$ of Players $I I$ is a function of values $\widetilde{b}_{i j}$, denoted by $v=v\left(\widetilde{b}_{i j}\right)$. At the same time, $y^{*} \in S^{n}$ of Players $I$ is also a function of values $\widetilde{b}_{i j}$, denoted by $y^{*}=y^{*}\left(\widetilde{b}_{i j}\right)$. we can get the following theorem. 
Theorem 4 For any a given game $(B G F P)$ model, if $\left(\widetilde{a}_{i j}, \widetilde{b}_{i j}\right) \in \widetilde{A}_{i j} \times \widetilde{B}_{i j}(i=$ $1,2, \cdots, m ; j=1,2, \cdots, n)$, then $u=u\left(\widetilde{a}_{i j}\right)$ and $v=v\left(\widetilde{b}_{i j}\right)$ of Players $I$ and Players II are monotonic nondecreasing functions.

Proof. For any a given game $(B G F P)$ model, $\left(\widetilde{a}_{i j}, \widetilde{b}_{i j}\right) \in \widetilde{A}_{i j} \times \widetilde{B}_{i j}$ and $\left(\widetilde{a}_{i j}^{\prime}, \widetilde{b}_{i j}^{\prime}\right) \in$ $\widetilde{A}_{i j} \times \widetilde{B}_{i j}$. In terms of mixed strategies, we assume that $\left(x^{*}, y^{*}\right)$ and $\left(\left(x^{*}\right)^{\prime},\left(y^{*}\right)^{\prime}\right)$ are respective equilibrium solutions of fuzzy bi-matrix games based on fuzzy payoffs $\widetilde{A}_{i j} \times$ $\widetilde{B}_{i j}$ and $\widetilde{A}_{i j}^{\prime} \times \widetilde{B}_{i j}^{\prime}$, where $\widetilde{A}_{i j}=\left(\widetilde{a}_{i j}\right)_{m \times n}, \widetilde{B}_{i j}=\left(\widetilde{b}_{i j}\right)_{m \times n}, \widetilde{A}_{i j}^{\prime}=\left(\widetilde{a}_{i j}^{\prime}\right)_{m \times n}$ and $\widetilde{B}_{i j}^{\prime}=$ $\left(\widetilde{b}_{i j}^{\prime}\right)_{m \times n}$. If $\left(\widetilde{a}_{i j}, \widetilde{b}_{i j}\right) \preceq\left(\widetilde{a}_{i j}^{\prime}, \widetilde{b}_{i j}^{\prime}\right)$, by Definition 1 , we have that $\left[\widetilde{a}_{i j}\right]^{\alpha} \leq\left[\widetilde{a}_{i j}^{\prime}\right]^{\alpha}$ and $\left[\widetilde{b}_{i j}\right]^{\alpha} \leq\left[\widetilde{b}_{i j}^{\prime}\right]^{\alpha}$. That is,

$$
\left(\widetilde{a}_{i j}\right)_{L}(\alpha) \leq\left(\widetilde{a}_{i j}^{\prime}\right)_{L}(\alpha), \quad\left(\widetilde{a}_{i j}\right)_{R}(\alpha) \leq\left(\widetilde{a}_{i j}^{\prime}\right)_{R}(\alpha)
$$

and

$$
\left(\widetilde{b}_{i j}\right)_{L}(\alpha) \leq\left(\widetilde{b}_{i j}^{\prime}\right)_{L}(\alpha), \quad\left(\widetilde{b}_{i j}\right)_{R}(\alpha) \leq\left(\widetilde{b}_{i j}^{\prime}\right)_{R}(\alpha)
$$

Thus, we have

$$
\begin{aligned}
& \sum_{i=1}^{m} \sum_{j=1}^{n}\left(x_{i}\right)^{*}\left(\widetilde{a}_{i j}\right)_{L}(\alpha)\left(y_{j}\right)^{*} \leq \sum_{i=1}^{m} \sum_{j=1}^{n}\left(x_{i}\right)^{*}\left(\widetilde{a}_{i j}^{\prime}\right)_{L}(\alpha)\left(y_{j}\right)^{*}, \\
& \sum_{i=1}^{m} \sum_{j=1}^{n}\left(x_{i}\right)^{*}\left(\widetilde{a}_{i j}\right)_{R}(\alpha)\left(y_{j}\right)^{*} \leq \sum_{i=1}^{m} \sum_{j=1}^{n}\left(x_{i}\right)^{*}\left(\widetilde{a}_{i j}^{\prime}\right)_{R}(\alpha)\left(y_{j}\right)^{*}, \\
& \sum_{i=1}^{m} \sum_{j=1}^{n}\left(x_{i}\right)^{*}\left(\widetilde{b}_{i j}\right)_{L}(\alpha)\left(y_{j}\right)^{*} \leq \sum_{i=1}^{m} \sum_{j=1}^{n}\left(x_{i}\right)^{*}\left(\widetilde{b}_{i j}^{\prime}\right)_{L}(\alpha)\left(y_{j}\right)^{*}
\end{aligned}
$$

and

$$
\sum_{i=1}^{m} \sum_{j=1}^{n}\left(x_{i}\right)^{*}\left(\widetilde{b}_{i j}\right)_{R}(\alpha)\left(y_{j}\right)^{*} \leq \sum_{i=1}^{m} \sum_{j=1}^{n}\left(x_{i}\right)^{*}\left(\widetilde{b}_{i j}^{\prime}\right)_{R}(\alpha)\left(y_{j}\right)^{*} .
$$

That is,

$$
\sum_{i=1}^{m} \sum_{j=1}^{n}\left(x_{i}\right)^{*} \widetilde{a}_{i j}\left(y_{j}\right)^{*} \preceq \sum_{i=1}^{m} \sum_{j=1}^{n}\left(x_{i}\right)^{*} \widetilde{a}_{i j}^{\prime}\left(y_{j}\right)^{*}
$$

and

$$
\sum_{i=1}^{m} \sum_{j=1}^{n}\left(x_{i}\right)^{*} \widetilde{b}_{i j}\left(y_{j}\right)^{*} \preceq \sum_{i=1}^{m} \sum_{j=1}^{n}\left(x_{i}\right)^{*} \widetilde{b}_{i j}^{\prime}\left(y_{j}\right)^{*} .
$$

Since in terms of mixed strategies, $\left(\left(x^{*}\right)^{\prime},\left(y^{*}\right)^{\prime}\right)$ is an equilibrium solution of fuzzy bi-matrix game based on fuzzy payoffs $\widetilde{A}_{i j}^{\prime} \times \widetilde{B}_{i j}^{\prime}$, we get

$$
\sum_{i=1}^{m} \sum_{j=1}^{n}\left(x_{i}\right)^{*} \widetilde{a}_{i j}^{\prime}\left(y_{j}\right)^{*} \preceq \sum_{i=1}^{m} \sum_{j=1}^{n}\left(\left(x_{i}\right)^{*}\right)^{\prime} \widetilde{a}_{i j}^{\prime}\left(\left(y_{j}\right)^{*}\right)^{\prime},
$$


and

$$
\sum_{i=1}^{m} \sum_{j=1}^{n}\left(x_{i}\right)^{*} \widetilde{b}_{i j}^{\prime}\left(y_{j}\right)^{*} \preceq \sum_{i=1}^{m} \sum_{j=1}^{n}\left(\left(x_{i}\right)^{*}\right)^{\prime} \widetilde{b}_{i j}^{\prime}\left(\left(y_{j}\right)^{*}\right)^{\prime},
$$

Therefore, combining with (19), (20), (21) and (22), we get

$$
\sum_{i=1}^{m} \sum_{j=1}^{n}\left(x_{i}\right)^{*} \widetilde{a}_{i j}\left(y_{j}\right)^{*} \preceq \sum_{i=1}^{m} \sum_{j=1}^{n}\left(\left(x_{i}\right)^{*}\right)^{\prime} \widetilde{a}_{i j}^{\prime}\left(\left(y_{j}\right)^{*}\right)^{\prime},
$$

and

$$
\sum_{i=1}^{m} \sum_{j=1}^{n}\left(x_{i}\right)^{*} \widetilde{b}_{i j}\left(y_{j}\right)^{*} \preceq \sum_{i=1}^{m} \sum_{j=1}^{n}\left(\left(x_{i}\right)^{*}\right)^{\prime} \widetilde{b}_{i j}^{\prime}\left(\left(y_{j}\right)^{*}\right)^{\prime},
$$

That is,

$$
\left(x^{*}\right)^{T} \widetilde{A} y^{*} \preceq\left(\left(x^{*}\right)^{\prime}\right)^{T} \widetilde{A}^{\prime}\left(y^{*}\right)^{\prime}
$$

and

$$
\left(x^{*}\right)^{T} \widetilde{B} y^{*} \preceq\left(\left(x^{*}\right)^{\prime}\right)^{T} \widetilde{B}^{\prime}\left(y^{*}\right)^{\prime} .
$$

Then $u=u\left(\widetilde{a}_{i j}\right)$ and $v=v\left(\widetilde{b}_{i j}\right)$ of Players $I$ and Players $I I$ are monotonic nondecreasing functions.

Inspired by $[10,16]$, the values of two Players in terms of game BGIVFP model are closed interval, denoted by $[\underline{u}(\alpha), \bar{u}(\alpha)]=\left[u\left(\left(\widetilde{a}_{i j}\right)_{L}(\alpha)\right), u\left(\left(\widetilde{a}_{i j}\right)_{R}(\alpha)\right)\right]$ and $[\underline{v}(\alpha), \bar{v}(\alpha)]=$ $\left[v\left(\left(\widetilde{b}_{i j}\right)_{L}(\alpha)\right), v\left(\left(\widetilde{b}_{i j}\right)_{R}(\alpha)\right)\right]$, respectively. Where $\underline{u}(\alpha)=u\left(\left(\widetilde{a}_{i j}\right)_{L}(\alpha)\right), \bar{u}(\alpha)=u\left(\left(\widetilde{a}_{i j}\right)_{R}(\alpha)\right)$ $\underline{v}(\alpha)(\alpha)=v\left(\left(\widetilde{b}_{i j}\right)_{L}(\alpha)\right), \bar{v}(\alpha)=v\left(\left(\widetilde{b}_{i j}\right)_{R}(\alpha)\right)$ By the proof of Theorem $4, \underline{u}(\alpha)$ and $\underline{v}(\alpha)$ are the lower bounds values of Players $I$ and Players $I I$. Correspondingly, $\underline{x}(\alpha)=$ $\left(x\left(\left(\widetilde{a}_{i j}\right)_{L}(\alpha)\right)\right.$ and $\left.\underline{y}(\alpha)=y\left(\left(\widetilde{b}_{i j}\right)_{L}(\alpha)\right)\right)$ are equilibrium solutions of Players $I$ and Players $I I$.

By Theorem 1, we have that $\underline{x}(\alpha)$ and $\underline{y}(\alpha)$ are equilibrium solutions of Players $I$ and Players $I I$ if and only if, $(\underline{x}(\alpha), \underline{y}(\alpha))$ is an optimal solution the following problem (NLP2) model.

$$
\begin{gathered}
(N L P 2) \max \left\{\sum_{i=1}^{m} \sum_{j=1}^{n} \underline{x}_{i}(\alpha)\left(\widetilde{a}_{i j}\right)_{L}(\alpha) \underline{y}_{j}(\alpha)+\sum_{i=1}^{m} \sum_{j=1}^{n} \underline{x}_{i}(\alpha)\left(\widetilde{b}_{i j}\right)_{L}(\alpha) \underline{y}_{j}(\alpha)-\underline{u}(\alpha)-\underline{v}(\alpha)\right\} \\
\text { subject to } \quad \sum_{j=1}^{n}\left(\widetilde{a}_{i j}\right)_{L}(\alpha) \underline{y}_{j}(\alpha) \leq \underline{u}(\alpha)(i=1,2, \cdots, m), \\
\\
\sum_{i=1}^{m}\left(\widetilde{b}_{i j}\right)_{L}(\alpha) \underline{x}_{i}(\alpha) \leq \underline{v}(\alpha), \quad(j=1,2, \cdots, n), \\
\sum_{i=1}^{m} \underline{x}_{i}=1, \\
\sum_{j=1}^{n} \underline{y}_{j}=1, \\
\underline{x}_{i} \geq 0, \underline{y}_{j} \geq 0, \\
\alpha \in[0,1] .
\end{gathered}
$$


Similarly, by the proof of Theorem $4, \bar{u}(\alpha)$ and $\bar{v}(\alpha)$ are the upper bounds values of Players $I$ and Players $I I$. Correspondingly, $\bar{x}(\alpha)=\left(x\left(\left(\widetilde{a}_{i j}\right)_{R}(\alpha)\right)\right.$ and $\bar{y}(\alpha)=$ $\left.y\left(\left(\widetilde{b}_{i j}\right)_{R}(\alpha)\right)\right)$ are equilibrium solutions of Players $I$ and Players $I I$.

By Theorem 1, we have that $\bar{x}(\alpha)$ and $\bar{y}(\alpha)$ are equilibrium solutions of Players $I$ and Players $I I$ if and only if, $(\bar{x}(\alpha), \bar{y}(\alpha))$ is an optimal solution the following problem (NLP3) model.

$$
\begin{gathered}
(N L P 3) \max \left\{\sum_{i=1}^{m} \sum_{j=1}^{n} \bar{x}_{i}(\alpha)\left(\widetilde{a}_{i j}\right)_{R}(\alpha) \bar{y}_{j}(\alpha)+\sum_{i=1}^{m} \sum_{j=1}^{n} \bar{x}_{i}(\alpha)\left(\widetilde{b}_{i j}\right)_{R}(\alpha) \bar{y}_{j}(\alpha)-\bar{u}(\alpha)-\bar{v}(\alpha)\right\} \\
\text { subject to } \quad \sum_{j=1}^{n}\left(\widetilde{a}_{i j}\right)_{R}(\alpha) \bar{y}_{j}(\alpha) \leq \bar{u}(\alpha) \quad(i=1,2, \cdots, m), \\
\sum_{i=1}^{m}\left(\widetilde{b}_{i j}\right)_{R}(\alpha) \bar{x}_{i}(\alpha) \leq \bar{v}(\alpha), \quad(j=1,2, \cdots, n), \\
\sum_{i=1}^{m} \bar{x}_{i}=1, \\
\sum_{j=1}^{n} \bar{y}_{j}=1, \\
\bar{x}_{i} \geq 0, \bar{y}_{j} \geq 0, \\
\alpha \in[0,1] .
\end{gathered}
$$

\section{Example}

In order to illustrate the effectiveness and correctness of the obtained model. We now consider the following fuzzy bi-matrix game $(B G F P)$ model.

Example 1 For any a given game (BGFP) model, the fuzzy payoff matrixes of the Player I and Player II are taken as

$$
\widetilde{A}=\left(\begin{array}{cc}
(11.75,12.00,12.60) & (16.80,17.00,18.00) \\
(12.50,13.00,13.25) & (15.75,16.00,16.25)
\end{array}\right)
$$

and

$$
\widetilde{B}=\left(\begin{array}{cc}
(10.80,11.00,11.70) & (14.75,15.00,15.45) \\
(9.50,10.00,10.80) & (13.50,14.00,14.55)
\end{array}\right)
$$

respectively.

Now, we solve this problem with the above model. By using (1), (11) and (12), we obtain the following payoff matrixes based on interval value function of Player I and Player II

$$
I(\widetilde{A})=\left(\begin{array}{ll}
{[0.25 \alpha+11.75,-0.60 \alpha+12.60]} & {[0.20 \alpha+16.80,-1.00 \alpha+18.00]} \\
{[0.50 \alpha+12.50,-0.25 \alpha+13.25]} & {[0.25 \alpha+15.75,-0.25 \alpha+16.25]}
\end{array}\right)
$$


and

$$
I(\widetilde{B})=\left(\begin{array}{cc}
{[0.20 \alpha+10.80,-0.70 \alpha+11.70]} & {[0.25 \alpha+14.75,-0.45 \alpha+15.45]} \\
{[0.50 \alpha+9.50,-0.80 \alpha+10.80]} & {[0.50 \alpha+13.50,-0.55 \alpha+14.55]}
\end{array}\right)
$$

respectively. Thus now utilizing (27) and (28), we obtain the following problems $(N L P 4)$ and $(N L P 5)$.

$$
\begin{aligned}
& \text { max } \quad\left\{(0.45 \alpha+22.55) \underline{x}_{1} \underline{y}_{1}+(\alpha+22) \underline{x}_{2} \underline{y}_{1}+(0.45 \alpha+31.55) \underline{x}_{1} \underline{y}_{2}\right. \\
&+\left.(0.75 \alpha+29.25) \underline{x}_{2} \underline{y}_{2}-\underline{u}(\alpha)-\underline{v}(\alpha)\right\} \\
& \text { subject to } \quad(0.25 \alpha+11.75) \underline{y}_{1}+(0.20 \alpha+16.80) \underline{y}_{2} \leq \underline{u}(\alpha)(0.50 \alpha+12.50) \underline{y}_{1}+(0.25 \alpha+15.75) \underline{y}_{2} \leq \underline{u}(\alpha) \\
&(0.20 \alpha+10.80) \underline{x}_{1}+(0.50 \alpha+9.50) \underline{x}_{2} \leq \underline{v}(\alpha) \\
&(0.25 \alpha+14.75) \underline{x}_{1}+(0.50 \alpha+13.50) \underline{x}_{2} \leq \underline{v}(\alpha) \\
& \underline{x}_{1}+\underline{x}_{2}=1, \underline{y}_{1}+\underline{y}_{2}=1 \\
& \underline{x}_{1} \geq 0, \underline{x}_{2} \geq 0, \underline{y}_{1} \geq 0, \underline{y}_{2} \geq 0 \\
& \alpha \in[0,1] .
\end{aligned}
$$

and

$(N L P 5) \quad \max \quad\left\{(-1.30 \alpha+24.3) \bar{x}_{1} \bar{y}_{1}+(-1.05 \alpha+24.05) \bar{x}_{2} \bar{y}_{1}+(-1.45 \alpha+33.45) \bar{x}_{1} \bar{y}_{2}\right.$

$$
\left.+(-0.8 \alpha+30.8) \bar{x}_{2} \bar{y}_{2}-\bar{u}(\alpha)-\bar{v}(\alpha)\right\}
$$

subject to $\quad(-0.60 \alpha+12.60) \bar{y}_{1}+(-1.00 \alpha+18.00) \bar{y}_{2} \leq \bar{u}(\alpha)$,

$(-0.25 \alpha+13.25) \bar{y}_{1}+(-0.25 \alpha+16.25) \bar{y}_{2} \leq \bar{u}(\alpha)$,

$(-0.70 \alpha+11.70) \bar{x}_{1}+(-0.80 \alpha+10.80) \bar{x}_{2} \leq \bar{v}(\alpha)$,

$(-0.45 \alpha+15.45) \bar{x}_{1}+(-0.55 \alpha+14.55) \bar{x}_{2} \leq \bar{v}(\alpha)$,

$\bar{x}_{1}+\bar{x}_{2}=1, \bar{y}_{1}+\bar{y}_{2}=1$,

$\bar{x}_{1} \geq 0, \bar{x}_{2} \geq 0, \bar{y}_{1} \geq 0, \bar{y}_{2} \geq 0$,

$\alpha \in[0,1]$.

Utilizing the Lingo software, particularly, let $\alpha^{*}=0.2$, we can get that $\left(\underline{x}^{*}, \underline{y}^{*}, \underline{u}^{*}, \underline{v}^{*}\right)$ and $\left(\bar{x}^{*}, \bar{y}^{*}, \bar{u}^{*}, \bar{v}^{*}\right)$ are optimal solutions of problems $(N L P 4)$ and $(N L P 5)$, respectively. Where $\underline{x}^{*}=\left(\underline{x}_{1}^{*}=0.32, \underline{x}_{2}^{*}=0.68\right), \underline{y}^{*}=\left(\underline{y}_{1}^{*}=0.24, \underline{y}_{2}^{*}=0.76\right), \underline{u}^{*}=\underline{u}\left(\alpha^{*}\right)=$ $15.63, \underline{v}^{*}=\underline{v}\left(\alpha^{*}\right)=11.26, \bar{x}^{*}=\left(\bar{x}_{1}^{*}=0.37, \bar{x}_{2}^{*}=0.63\right), \bar{y}^{*}=\left(\bar{y}_{1}^{*}=0.30, \bar{y}_{2}^{*}=\right.$ $0.70), \bar{u}^{*}=\bar{u}\left(\alpha^{*}\right)=16.20, \bar{v}^{*}=\bar{v}\left(\alpha^{*}\right)=14.78$.

Thus, we obtain that $\underline{u}^{*}=15.63$ and $\underline{v}^{*}=11.26$ are the lower bounds values of Players $I$ and Players II. Correspondingly, $\underline{x}^{*}=\left(\underline{x}_{1}^{*}=0.32, \underline{x}_{2}^{*}=0.68\right)$ and $\underline{y}^{*}=\left(\underline{y}_{1}^{*}=\right.$ $\left.0.24, \underline{y}_{2}^{*}=0.76\right)$ are equilibrium solutions of Players $I$ and Players $I I$.

Similarly, $\bar{u}^{*}=16.20$ and $\bar{v}^{*}=14.78$ are the upper bounds values of Players $I$ and Players $I I$. Correspondingly, $\bar{x}^{*}=\left(\bar{x}_{1}^{*}=0.37, \bar{x}_{2}^{*}=0.63\right)$ and $\bar{y}^{*}=\left(\bar{y}_{1}^{*}=0.30, \bar{y}_{2}^{*}=\right.$ $0.70)$ are equilibrium solutions of Players $I$ and Players $I I$. 


\section{Conclusion}

This article has presented the bi-matrix games with crisp parametric payoffs based on interval value function approach. It is shown that the equilibrium solution of the game model can be changed into the pair of optimal solutions of the non-linear optimization problem. In recent years, more and more researchers have extensively studied the bi-matrix game model under the environment of intuitionistic fuzzy and entropy $[11,12,18,22,23,25,26]$. Further studies involving the game model of intuitionistic fuzzy entropy environment are expected to be discussed. And we will use other more effective ways to study the bi-matrix game problem in the future.

\section{Acknowledgements}

This work was supported by The National Natural Science Foundations of China (Grant no. 11671001 and 61472056).

\section{References}

[1] Basar, T.; Olsder, G.J. Dynamic Noncooperative Game Theory. Second Edition. Academic, San Diego, 1995.

[2] Bector, C. R.; Chandra, S. Fuzzy mathematical programming and fuzzy matrix games. Berlin Heidelberg: Springer-Verlag, 2005.

[3] Blackwell, D. An analog of the minimax theorem for vector payoff. Pac. J. Math., 1956, $1-8$.

[4] Chalco-Canoa, Y.; Rufián-Lizana, A.; Román-Flores,H.; Osuna-Gómez. R. A note on generalized convexity for fuzzy mappings through a linear ordering. Fuzzy Sets Syst., 2013, $231,70-83$.

[5] Chakeri, A.; Sadati, N.; Sharifian, S. Fuzzy Nash equilibrium in fuzzy games using ranking fuzzy numbers. In Proceedings of the 2010 IEEE International Conference on Fuzzy Systems (FUZZ), Barcelona, Spain, 18-23 July 2010; pp. 1-5.

[6] Chakeri, A.; Sheikholeslam, F. Fuzzy Nash equilibriums in crisp and fuzzy games. IEEE Trans. Fuzzy Syst. 2013, 21, 171-176.

[7] Chakeri, A.; Sadati, N.; Dumont, G.A. Nash Equilibrium Strategies in Fuzzy Games. Game Theory Relaunched; InTech: Rijeka, Croatia, 2013; doi:10.5772/54677.

[8] Chakeri, A.; Habibi, J.; Heshmat, Y. Fuzzy type-2 Nash equilibrium. In Proceedings of the 2008 International Conference on Computational Intelligence for Modelling Control and Automation, Vienna, Austria, 10-12 December 2008; pp. 398-402.

[9] Chakeri, A.; Dariani, A.N.; Lucas, C. How can fuzzy logic determine game equilibriums better? In Proceedings of the 4th International IEEE Conference on Intelligent Systems (IS08), Varna, Bulgaria, 6-8 September 2008; pp. 251-256.

[10] Davvaz, B.; Khan, A.; Sarmin. N.H. More general forms of interval valued fuzzy filters of ordered semigroups. Int. J. Fuzzy Syst., 2013, 15, 110-126. 
[11] Das, C. B.; Roy, S. K. Fuzzy based GA to multi-objective entropy bimatrix game. Opsearch, 2013, 50, 125-140.

[12] DeLuca, A.; Termini, S. A definition of a nonprobabilistic entropy in the setting of fuzzy sets theory. Inf. Control, 1972, 20, 301-312.

[13] Diamond, P.; Kloeden, P. Metric spaces of fuzzy sets. Fuzzy Sets Syst., 1990, 35, 241-249.

[14] Dubois, D.; Prade, H. Fuzzy sets and systems-theory and application. Academic Press, New York, 1980.

[15] Hladk, M. Interval valued bimatrix games. Kybernetika -Praha-, 2010, 3, 435-446.

[16] Fei, W.; Li, D. F. Bilinear Programming Approach to Solve Interval Bimatrix Games in Tourism Planning Management, Int. J. Fuzzy Syst., 2016, 18, 504-510.

[17] Li, C. Characterization of the Equilibrium Strategy of Fuzzy Bimatrix Games Based on Fuzzy Variables. J. Appl. Math., 2012, 3, 331-353.

[18] Li, D.F. Decision and Game Theory in Management with Intuitionistic Fuzzy Sets. Studies in Fuzziness and Soft Computing 308, Springer-Verlag Berlin Heidelberg, 2014.

[19] Li, L.; Liu, S.Y.; Zhang, J.K. On fuzzy generalized convex mappings and optimality conditions for fuzzy weakly univex mappings. Fuzzy Sets Syst., 2015, 280, 107-132.

[20] Maeda, T. On characterization of equilibirum strategy of the bimatrix game with fuzzy payoff. J. Math. Anal. Appl., 2000, 251, 885-896.

[21] Mangasarian, O.L.; Stone, H. Two person non zero sum game and quadratic programming. J. Math. Anal. Appl., 1964, 9, 348-355.

[22] Nan, J.X.; Zhang, M.J.; Li, D.F. Intuitionistic fuzzy programming models for matrix games with payoffs of trapezoidal intuitionistic fuzzy numbers. Int. J. Fuzzy Syst., 2014, $16,444-456$.

[23] Nayak, P.K.; Pal, M. Bimatrix games with intiutionstic fuzzy goals, Iran. J. Fuzzy Syst., 2010, 7, 65-79.

[24] Panigrahi, M.; Panda, G.; Nanda, S. Convex fuzzy mapping with differentiability and its application in fuzzy optimization. Eur. J. Oper. Res., 2008, 185, 47-62.

[25] Roy, S. K.; Biswal, M. P.; Tiwari, R.N. An approach to multi-objective bimatrix games for Nash equilibrium solutions. Ric. Oper., 2001, 30, 56-63.

[26] Roy, S. K. Fuzzy programming approach to two-person multicriteria bimatrix games, J. Fuzzy Math., 2007, 15, 141-153.

[27] Sharifian, S.; Chakeri, A.; Sheikholeslam, F. Linguisitc representation of Nash equilibriums in fuzzy games. In Proceedings of the IEEE 2010 Annual Meeting of the North American Fuzzy Information Processing Society (NAFIPS), Toronto, ON, Canada, 12-14 July 2010; pp. 1-6.

[28] Syau, Y.R.; Stanley Lee, E. Fuzzy Weirstrass theorem and convex fuzzy mappings. Comput. Math. Appl., 2006, 51, 1741-1750.

[29] Syau, Y.R.; Stanley Lee, E. Preinvexity and F1-convexity of fuzzy mappings through a linear ordering, Comput. Math. Appl., 2006, 51, 405-418. 
[30] Wang, G.X.; Wu, C.X. Directional derivatives and subdifferential of convex fuzzy mappings and application in convex fuzzy programming. Fuzzy Sets Syst. 2003, 138, 559-591.

[31] Vidyottama, V.; Chandra S.; Bector, C.R. Bi-matrix game with fuzzy goals and fuzzy payoffs. Fuzzy Optim. Decis. Ma., 2004, 3, 327-344.

[32] Vijay, V.; Mehra, A.; Chandra S.; Bector, C.R. Fuzzy matrix games via a fuzzy relation approach. Fuzzy Optim. Decis. Ma., 2007, 6, 299-314.

[33] Larbani, M. Solving bi-matrix games with fuzzy payoffs by introducing nature as a third player, Fuzzy Sets Syst., 2009, 160, 657-666.

[34] Zadeh, L. A. Fuzzy sets, Inf. Control, 1965, 8, 338-353.

[35] Zimmermann, H. J. Fuzzy programming and linear programming with several objective functions, Fuzzy Sets Syst., 1978, 1, 45-55. 